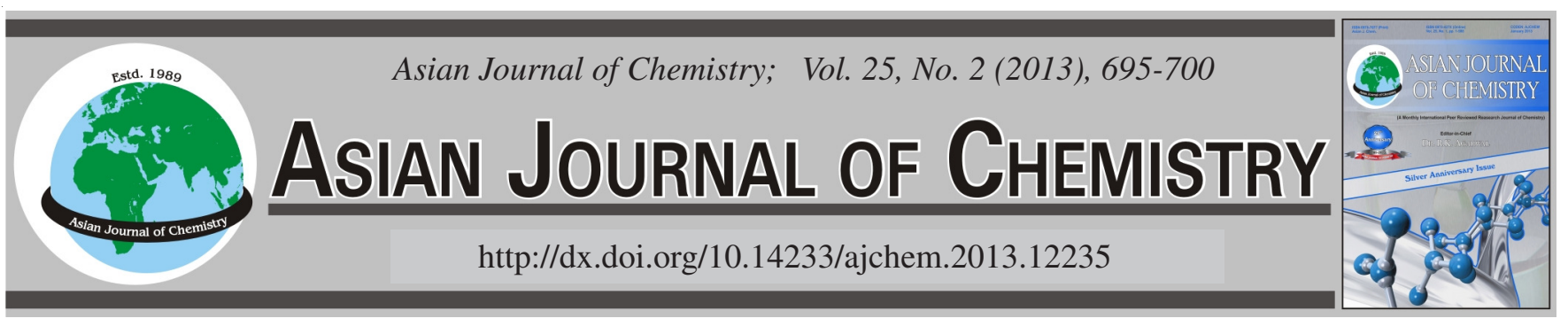

\title{
Effect of Nitrogen Gas Cold Plasma on Cotton Fabric Dyed with Reactive Dyes
}

\author{
H. AKBARPOUR ${ }^{1, *}$, A. RASHIDI ${ }^{2}$, M.E. YAZDANSHENAS ${ }^{3}$ and H. TAYEBI ${ }^{4}$
}

${ }^{1}$ Young Researchers Club, Yazd Branch, Islamic Azad University, Yazd, Iran

${ }^{2}$ Department of Textile Engineering, Science and Research Branch, Islamic Azad University, Tehran, Iran

${ }^{3}$ Department of Textile Engineering, Yazd Branch, Islamic Azad University, Yazd, Iran

${ }^{4}$ Department of Textile Engineering, Qaemshahr Branch, Islamic Azad University, Qaemshahr, Iran

*Corresponding author: E-mail: hamid.akbarpour578@gmail.com

(Received: 18 August 2011;

Accepted: 14 August 2012)

AJC-11959

In this paper, a bleached well cotton sample was dyed with cold and hot reactive dyes and subsequently, the dyed samples were exposed
to plasma condition containing nitrogen gas at 1 and 5 min of plasma exposure time. The effect of plasma on surface morphology was
studied by scanning electronic microscope. The K/S and $\mathrm{L}^{*} \mathrm{a}^{*} \mathrm{~b}^{*}$ values were evaluated using a reflective spectrophotometer and
consequently, the experiments showed that the sample dyed with reactive yellow 3 and reactive yellow 105 enjoys dye fastness which
decreases after being washed due to the increase in the operation time of plasma. In addition, the increase in plasma operation time at
constant pressure would increase the destructing effect on the surface morphology of samples dyed with hot and cold reactive. Finally, the
Fourier transform Infra-red spectroscopy test showed the effect of plasma operation on determining the dye fastness and the change in
existent functional groups in fabric after being washed.

Key Words: Cotton fabric, Nitrogen cold plasma, Reflective spectrophotometer, Scanning electronic microscope, Reactive dyes, | Fourier transform infrared spectroscopy.

ᄂ - - - - - - - - - - - - - - - - - - - - - - - - - - - -

\section{INTRODUCTION}

The plasma cold gases are often used for refining polymers' surface and also recently for refining the textile cloths made from natural and artificial fibers ${ }^{1-3}$. The plasma operations could effectively change the features of polymer's surface such as water absorption, adhesion and surface energy. The operations done by different gas plasmas could widely change the water absorption from moderate hydrophilic to high hydrophilic. In fact, plasma, depending on the experiment conditions, could result in these four effects i.e., purification (removal of organic contaminations), corrosion (removal of polymer materials and polymer destruction), create cross link bond (free radicals formation and make macromolecules branching), Forming new level of functional groups at a high depth of $10 \mathrm{~nm}^{1,4,5}$. Advantages of plasma operation are as follows; it modifies the farthest outer part of a thin surface layer while keeping the mass particulars unchanged, has the lowest chemical consumption and the highest safety, does not produce sewage, has the smallest effect on the environment and in the ecology of the textile mills $^{6,7}$. Plasma operation with other noble gases results in the production of various functional groups. In this method, modifying the plasma operation, due to plasma's limited-like penetration, is not effective on surface features of polymers. This does not happen for fibers and fibrils because of their high penetration depth ${ }^{1,8}$. In the study of oxygen plasma effect on cotton fabrics' water absorption, done by researchers, the most proper operation was carried out by changing the gas pressure in a range of 1-100 bars $^{1,9}$. Processing polyester and wool fabrics with argon/helium cold plasma under the atmosphere pressure increases the wettability of fabrics, causes colour saturation when dying samples and brings about exhaustion. In addition, nylon and poly propylene fabrics are modified in atmosphere pressure plasma. It is proved that oxygen and hydrogen mass will increase in poly propylene fabrics with both helium plasma and helium/oxygen plasma ${ }^{6,10}$. Helium/oxygen plasma and air/ $\mathrm{O}_{2}$ are used for sizing PVA (poly vinyl alcohol) on cotton ${ }^{6,11}$. In silk fabrics, the cold plasma operation was applied for inkjet pigment print and researchers found that the inkjet print reflection on silk fabric was deeper and it showed a brighter colour compared to fabrics not treated with plasma ${ }^{6,12}$. Fibers are naturally not conductive and this results in static electricity and its long durability on fabrics. In many cases, high volumes of static electricity may produce sparks and cause fire. Nevertheless, the low percentage of amorphous and high percentage of crystal parts on polyester 
fabrics, cause low moisture content and consequently, poses many problems for polyester. Plasma method was used to modify the polyester surface to increase the moisture content and the electricity distribution speed in the air ${ }^{13}$. It was proved that the cold plasma operation results in an increase of hydrophilicity features and improves the dyeability of wool fabrics ${ }^{14-16}$.

\section{EXPERIMENTAL}

At first, before cold plasma operation, $48 \mathrm{~g}$ of bleached cotton fabric in $50 \mathrm{~cm} \times 50 \mathrm{~cm}$ dimension was chosen from BABAKAN Textile Company. Initially, the fabric was washed at $60{ }^{\circ} \mathrm{C}$ and for 15 min with hot water and LCF-123 Jintery Eco anionic detergent in order to remove fabric's contamination; then the fabric was dried in a dryer for $1 \mathrm{~h}$ at $80^{\circ} \mathrm{C}$ and dyed through two stages with cold and hot reactive dyes. The first dying stage, as shown in Fig. 1, was performed with $2 \%$ of cold dye, namely reactive yellow K-RN (reactive yellow 3 ), with molecular structure shown in Fig. 2. The second stage, as shown in Fig. 3, was performed and washed with $1 \%$ of hot reactive dye, namely reactive yellow $\mathrm{HE}-4 \mathrm{G}$ (reactive yellow $105)$; both of the hot and cold reactive dyes were supplied from Tehran Dye Research Center. The predetermined L:R for both stages is 20:1. Samples were treated with cold plasma in regard to the supplied samples.

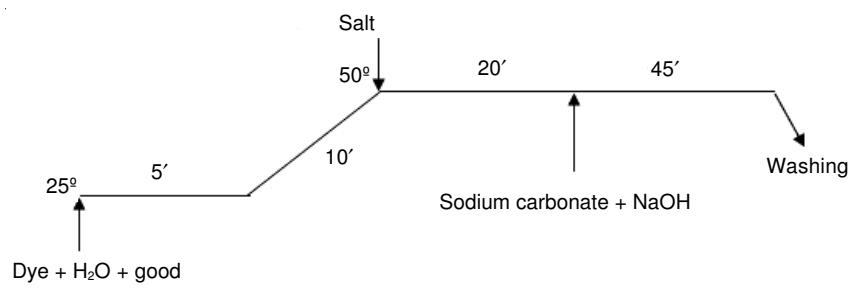

Fig. 1. Schematic graph showing the process of dying fabric with reactive yellow 3

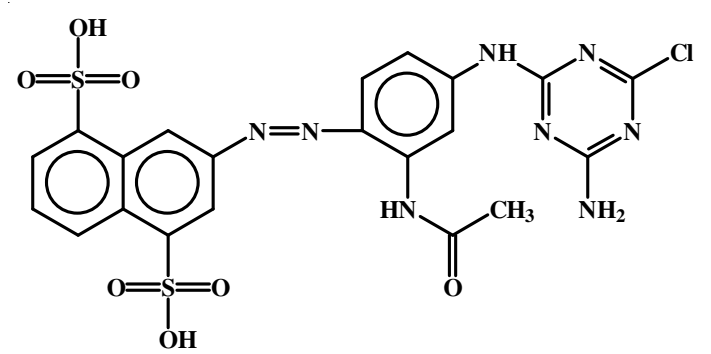

Fig. 2. Molecular structure of reactive dye named reactive yellow 3

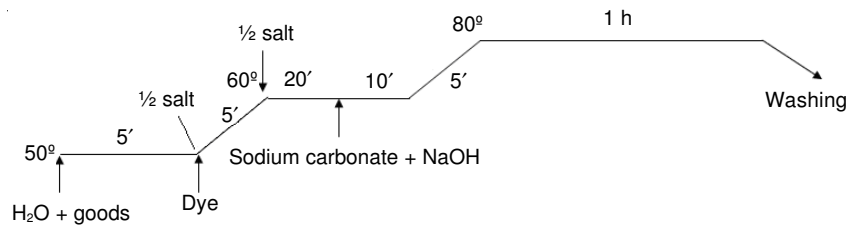

Fig. 3. Schematic graph showing the process of dying fabric with reactive yellow 105

Low temperature plasma treatments: At the first stage, four cold and hot reactive-dyed cotton fabric samples, each in $5 \mathrm{~cm} \times 5 \mathrm{~cm}$ dimension, were placed at vacuum condition for $1 \mathrm{~h}$; then the samples were exposed to nitrogen gas plasma condition, two of them for $1 \mathrm{~min}$ and the other 2 for $5 \mathrm{~min}$.
Plasma conditions were as follows: a system power of 100 watt with voltage of 500 volt was applied, the cathode was made from aluminum with $5 \times 10^{-2}$ torr pressure, the main current was 4 amperes and at last, the magnetic coil current was $200 \mathrm{~m}$ amperes. The schematic view of this system is shown in Fig. 4. The prepared samples were studied in next stages to evaluate the effect of plasma conditions on determining the $\mathrm{K} / \mathrm{S}$, Lab and the light reflection rate $(\mathrm{R} \%)$ and also to determine the washing fastness of fabrics.

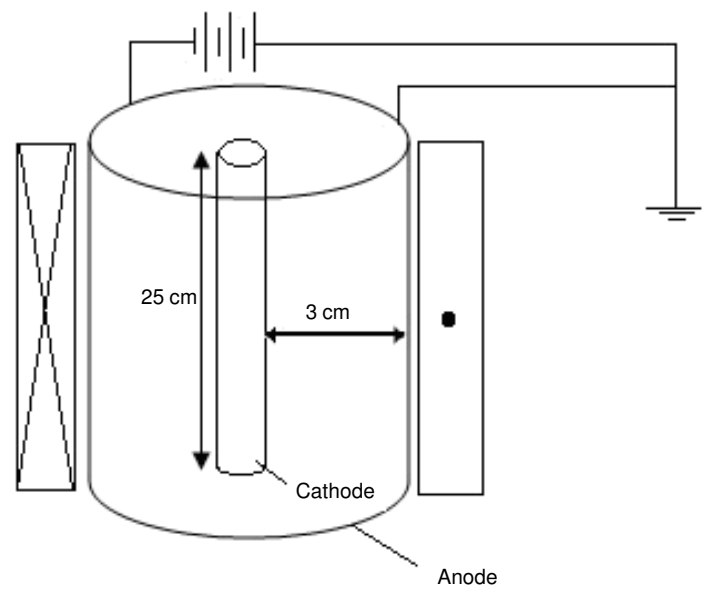

Fig. 4. Schematic view of the plasma system

Reflective spectrophotometer: The K/S, Lab and R \% rate of samples were measured by spectrophotometer ColourEye-7000A system with $\mathrm{d} / 8^{\circ}$ geometrical feature and UV D65 standard light source. This experiment was carried out to determine the colour fastness of the fabric resulted from being exposed to nitrogen gas cold plasma, before and after washing.

Scanning electronic microscope (SEM): $1 \mathrm{~cm} \times 1 \mathrm{~cm}$ pieces were cut out of experimented samples and were put in Sputter Coater machine to coat samples with a layer of gold. Then the untreated dyed and plasma-treated samples were scanned for 1 and 5 min using scanning electronic microscope, model LEO440 made in England with x 3000 magnification power, to determine the effect of plasma conditions on surface morphology of fabrics.

Washing fastness of the samples: In this experiment, samples weighing $5 \mathrm{~g}$, treated with plasma and dyed with cold and hot reactive, at exposure time of 1 and $5 \mathrm{~min}$, were washed 5 times for $0.5 \mathrm{~h}$ at $80^{\circ} \mathrm{C}$ heat in a soap bath containing $4 \mathrm{~g} / \mathrm{L}$ sodium hydroxide and $2 \mathrm{~g} / \mathrm{L}$ anionic soap and with 40/1 ratio of bath mass on weight of the fabric. Then, samples were dried in a dryer for $1 \mathrm{~h}$ and the K/S, Lab and \% $\mathrm{R}$ rate of washed samples were measured using a reflective spectrophotometer machine to determine colour fastness of fabrics.

Fourier transform Infrared spectroscopy (FTIR): In this study, each of the above samples was put in the machine jaw so as to experiment a complete level of samples. Then, samples were analyzed using OMNIC software. This experiment was operated in a machine named Thermo Nicollet 807 made in America, to prove the reliability of reflective spectrophotometer test in determining the colour and washing fastness of the fabric. 


\section{RESULTS AND DISCUSSION}

As shown in Fig. 5, the curves of the samples dyed with untreated and plasma treated reactive yellow 105 dyes, overlap each other at plasma exposure time of 1 and $5 \mathrm{~min}$. The plasmatic conditions show a low increscent in K/S rate. Table-1 show, display of difference samples that to be shown in Figs. 5-13.

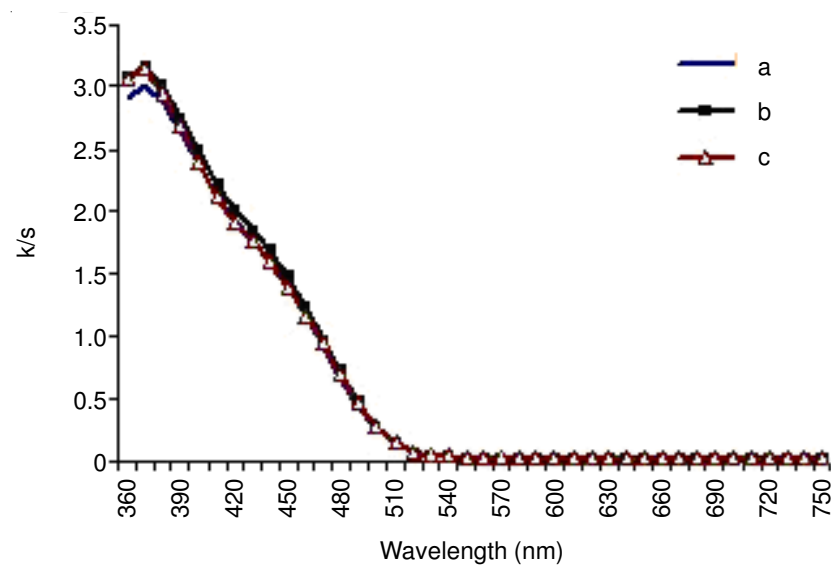

Fig. 5. K/S graph of samples dyed with untreated and plasma treated reactive yellow 105 within 1 and 5 minutes of plasma exposure time

\begin{tabular}{clcc}
\multicolumn{5}{c}{ TABLE-1 } \\
\multicolumn{5}{c}{$\begin{array}{c}\text { DISPLAY OF DIFFERENCE SAMPLES } \\
\text { THAT TO BE SHOWN IN FIGS. 5-13 }\end{array}$} \\
\hline Sample & \multicolumn{1}{c}{ Dyed with } & $\begin{array}{c}\text { Time of plasma } \\
\text { exposure time } \\
\text { before washing }\end{array}$ & $\begin{array}{c}\text { Time of } \\
\text { plasma } \\
\text { exposure time } \\
\text { after washing }\end{array}$ \\
\hline a & Reactive yellow 105 & Untreated & - \\
b & Reactive yellow 105 & 1 min & - \\
c & Reactive yellow 105 & 5 min & - \\
d & Reactive yellow 3 & Untreated & - \\
e & Reactive yellow 3 & 1 min & - \\
f & Reactive yellow 3 & 5 min & - \\
g & Reactive yellow 105 & - & 1 min \\
h & Reactive yellow 105 & - & 5 min \\
x & Reactive yellow 3 & - & 1 min \\
y & Reactive yellow 3 & - & 5 min \\
\hline
\end{tabular}

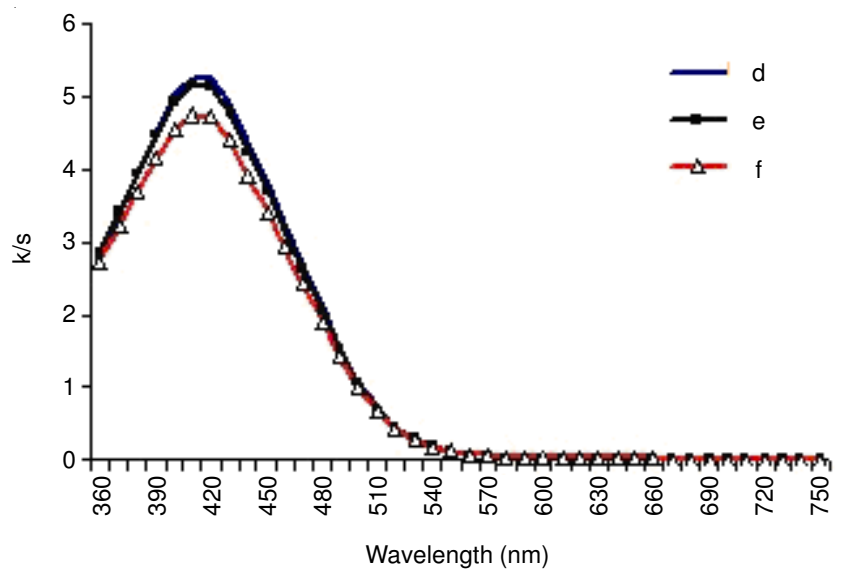

Fig. 6. K/S graph of samples dyed with untreated and plasma treated reactive yellow 3 at 1 and 5 min of plasma exposure time

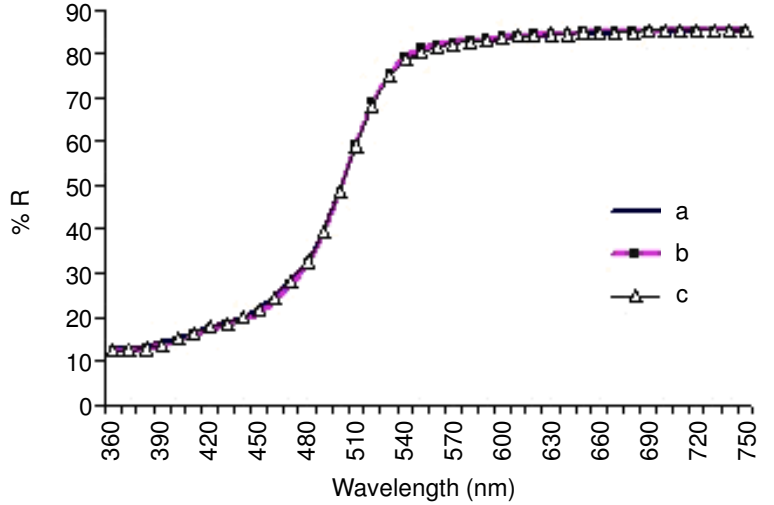

Fig. 7. R \% graph of samples dyed with untreated and plasma treated reactive yellow 105 at 1 and 5 min of plasma exposure time

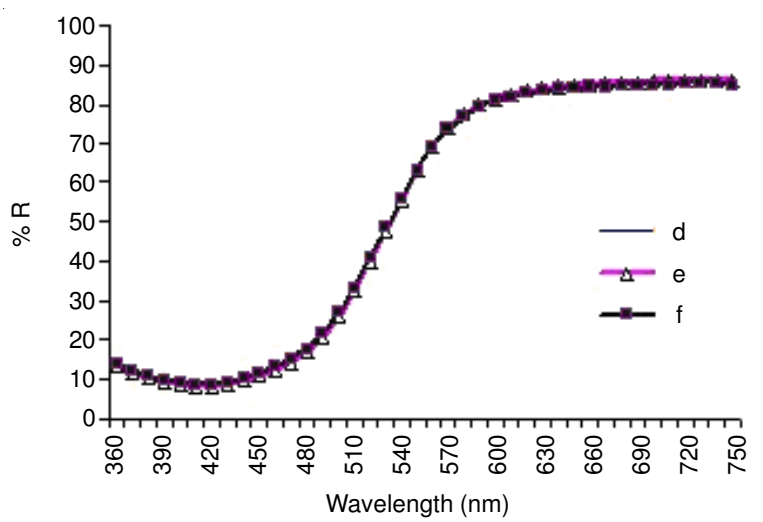

Fig. 8. R \% graph samples dyed with of untreated and plasma treated reactive yellow 3 with plasma at 1 and 5 min of plasma exposure time

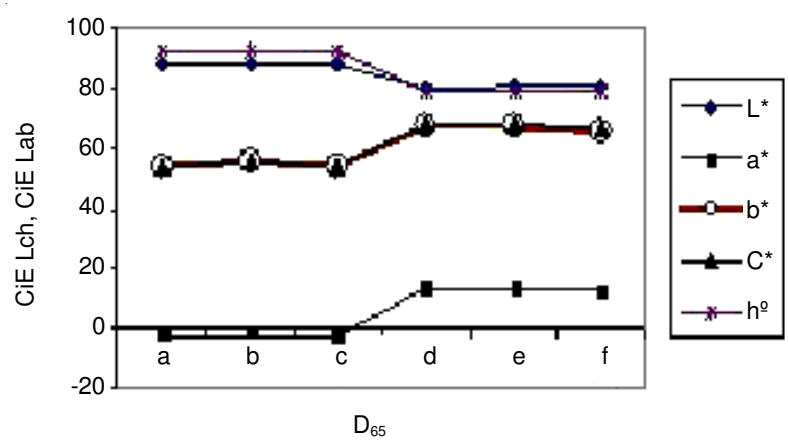

Fig. 9. Analogical graph related to triple-stimuli

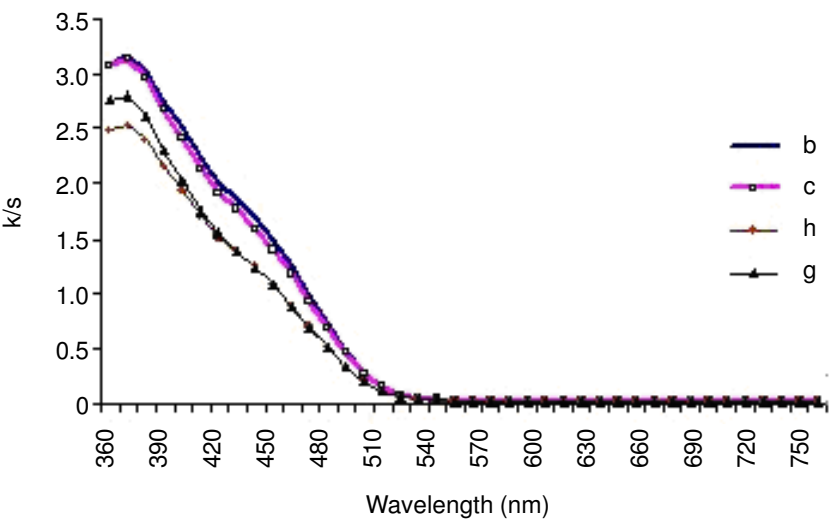

Fig. 10. K/S rate of samples dyed with plasma treated reactive yellow 105 at 1 and 5 min of plasma exposure time before and after washing 


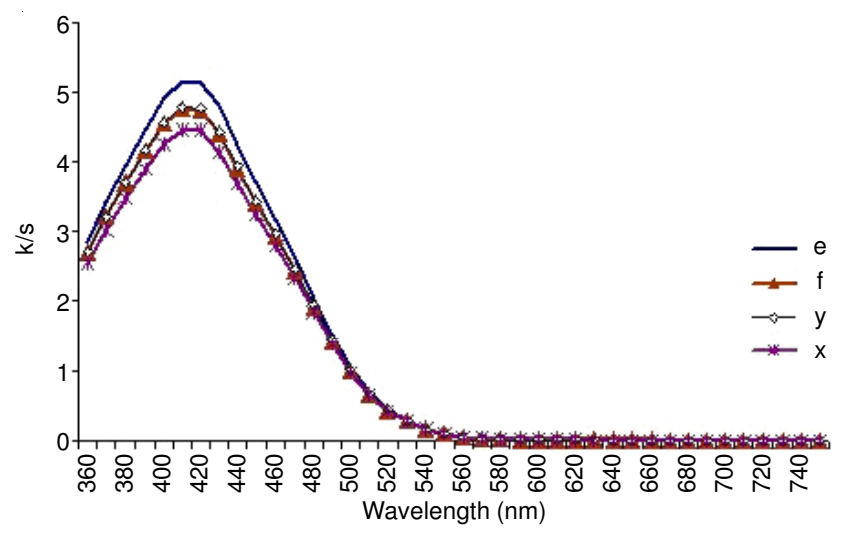

Fig. 11. K/S rate of samples dyed with plasma treated reactive yellow 3 at one and $5 \mathrm{~min}$ of plasma exposure time before and after washing

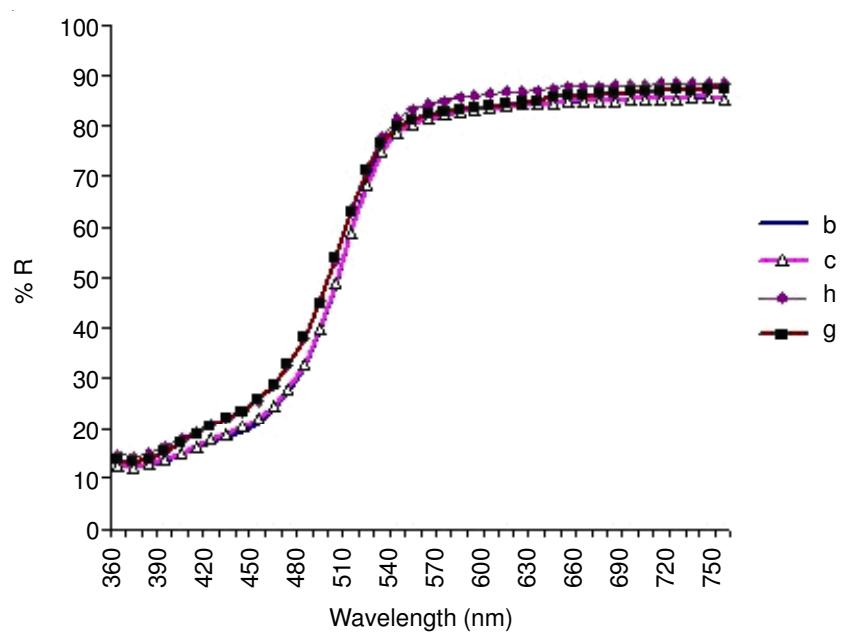

Fig. 12. R \% rates of samples dyed with plasma treated reactive yellow 105 at $1 \mathrm{~min}$ or $5 \mathrm{~min}$ of plasma exposure time before and after washing

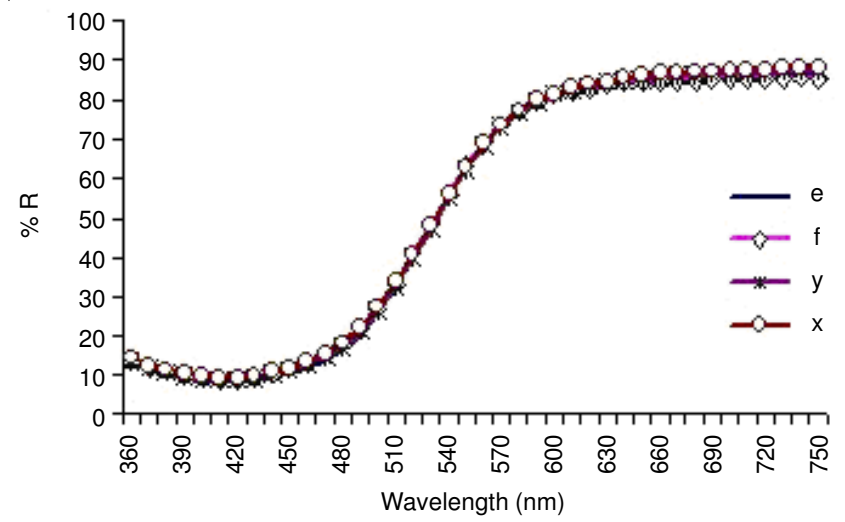

Fig. 13. R \% rates of samples dyed with plasma treated reactive yellow 3 at $1 \mathrm{~min}$ or $5 \mathrm{~min}$ of plasma exposure time before and after washing

As shown in Fig. 6, the peak decrease in the curve of sample dyed with reactive yellow 3, treated with plasma at plasma exposure time of 1 and 5 min, indicates that when the time of plasma operation increases, the decrease in the peak of the curve becomes more, although the difference in this range is slight for K/S coefficient. Thus, the plasma used for reactive yellow 3 doesn't have a significant effect on the results.

Reflective spectrum values of the experimented samples are graphically illustrated in Figs. 7 and 8 . These graphs show that the reflective spectrum curves were coincide with each other and plasmatic conditions don't have any effect on $\mathrm{R} \%$ rate of samples dyed with untreated and plasma treated reactive yellow 3 and reactive yellow 105. This confirms the results obtained from K/S curves.

The graph shown in Fig. 9 indicates the Lab rate, hue length and hue angle for D65 standard light source (illuminant). The $\mathrm{a}^{*}$ rate of standard D65, the standard daylight illuminant, is negative for the untreated samples dyed with reactive yellow 105 and plasma treated samples at 1 and 5 min of plasma exposure time $(1,2,3)$ compared to the untreated dyed sample with reactive yellow 3 and plasma treated sample at 1 and $5 \min (4,5,6)$. However, the a* rate was the same for each of 1 to 6 samples at standard light source; thus, it can be concluded that the plasma conditions don't affect the $\mathrm{a}^{*}$ rate. The $\mathrm{L}^{*}, \mathrm{c}^{*}$, $b^{*}$ and $h^{\circ}$ rates were also equal for all 6 samples; therefore, plasma doesn't have any effect on the Lab rate, hue length and hue angle $\left(\mathrm{h}^{\circ}\right)$, but shows the differences of Lab rate, hue length and hue angle between the sample dyed with reactive yellow 3 and the one dyed with reactive yellow 105 dyes.

Moreover, the results obtained from Tables 2 and 3, related to dye differences, proves that there isn't a significant difference between samples of each set, but there is a considerable difference between samples treated with cold and hot reactive, which is natural due to the change in the dye being used. Meanwhile, it is observed that the difference between the samples, not exposed to plasma $(1,4)$, was more than plasma treated samples. Thus, it can be concluded that plasma operation can somehow change the colour of samples. Tables 2 and 3 show the comparing values of $\Delta \mathrm{E}$ and triple-stimuli of samples dyed with hot and cold reactive at different times of plasma exposure.

TABLE-2

COMPARISON BETWEEN $\triangle$ E AND CIE OF SAMPLES DYED WITH PLASMA TREATED REACTIVE YELLOW 105

\begin{tabular}{cccc}
\hline \multicolumn{4}{c}{ Dyed with \% 1 of reactive yellow 105 } \\
\hline Time (plasma) & Untreated & $1 \mathrm{~min}$ & $5 \mathrm{~min}$ \\
\hline$\lambda_{\max }(\mathrm{nm})$ & 370 & 370 & 370 \\
$\mathrm{~K} / \mathrm{S}$ & 3.02 & 3.14 & 3.17 \\
$\mathrm{~L}^{*}$ & 88.24 & 88.27 & 88.09 \\
$\mathrm{~A}^{*}$ & -2.79 & -2.9 & -2.46 \\
$\mathrm{~b}^{*}$ & 54.29 & 55.61 & 54.08 \\
$\mathrm{C}^{*}$ & 54.36 & 55.68 & 54.13 \\
$\mathrm{~h}^{\text {o }}$ & 92.94 & 92.98 & 92.06 \\
$\Delta \mathrm{E}$ & - & 0.42 & 0.32 \\
\hline
\end{tabular}

TABLE-3

COMPARISON BETWEEN $\triangle$ E AND CIE OF SAMPLES DYED WITH PLASMA TREATED REACTIVE YELLOW 3 Dyed with $\% 2$ of reactive yellow 3

\begin{tabular}{cccc}
\multicolumn{4}{c}{ Dyed with \% 2 of reactive yellow 3 } \\
\hline Time (plasma) & Untreated & 1 min & $5 \mathrm{~min}$ \\
\hline$\lambda_{\max }(\mathrm{nm})$ & 5.28 & 5.17 & 4.77 \\
$\mathrm{~K} / \mathrm{S}$ & 80.51 & 80.62 & 80.74 \\
$\mathrm{~L}^{*}$ & 13.12 & 13.11 & 12.28 \\
$\mathrm{~A}^{*}$ & 67.59 & 67.09 & 65.34 \\
$\mathrm{~b}^{*}$ & 68.85 & 68.36 & 66.49 \\
$\mathrm{C}^{*}$ & 79.01 & 78.94 & 79.36 \\
$\mathrm{~h}^{\circ}$ & - & 0.51 & 2.41 \\
$\Delta \mathrm{E}$ & 5.28 & 5.17 & 4.77 \\
\hline
\end{tabular}


Scanning electronic microscope: Table- 4 shows the SEM images taken from the surface of dyed samples- untreated and treated with plasma at 1 and 5 min of plasma exposure timeby electronic microscope, indicates the difference in surface morphology. Results obtained from these images, show that the more the sample is exposed to plasmatic conditions of nitrogen gas, the more will be the effect of destructive conditions on it.

TABLE-4

IMAGES TAKEN FROM SAMPLES' SURFACE MORPHOLOGY USING SEM ELECTRONIC MICROSCOPE

\begin{tabular}{|c|c|c|c|}
\hline \multirow[b]{2}{*}{ Sample } & \multicolumn{3}{|c|}{ Time (Plasma) } \\
\hline & Untreated & $\begin{array}{c}\text { Treated with } \\
\text { plasma at } 1 \mathrm{~min} \\
\text { of exposure time }\end{array}$ & $\begin{array}{l}\text { Treated with } \\
\text { plasma at } 5 \mathrm{~min} \\
\text { of exposure time }\end{array}$ \\
\hline $\begin{array}{c}\text { Dyed with } \\
1 \% \text { of } \\
\text { reactive } \\
\text { yellow } \\
105\end{array}$ & & & \\
\hline $\begin{array}{c}\text { Dyed with } \\
2 \% \text { of } \\
\text { reactive } \\
\text { yellow } 3\end{array}$ & & & \\
\hline
\end{tabular}

Washing fastness: As it can be seen in the graph shown in Fig. 10, the washing effect on K/S rate is more comparing to its condition before washing (curves 1 and 2); consequently, it could be understood that samples' dye fastness decreases after washing (K/S rate decreases).

Fig. 11 shows the K/S rate of samples dyed with plasma treated cold reactive at 1 and 5 min of plasma exposure time before and after washing. This figure also shows that a decrease in the K/S rate happens for curves 7 and 8, comparing to their before-washing condition, which is due to the washing effect. This decrease in K/S rate shows the decrease in colour fastness. In Figs. 12 and 13, it can be easily seen that washing had an insignificant effect on $\mathrm{R} \%$ rate such that the curves of samples, before and after washing, are rather coincident.

Also, Tables 5 and 6, in comparison to Tables 2 and 3, show that the $\mathrm{K} / \mathrm{S}, \mathrm{C}^{*}, \mathrm{~h}^{\mathrm{o}}$ values have decreased after washing, while $\mathrm{E}, \mathrm{a}^{*}$ and $\mathrm{b}^{*}$ have been significantly increased. This shows the effect of washing conditions after plasma exposure. In other words, the plasma operation causes a slight decrease in washing fastness can as seen in Figs. 10-13.

Fourier transform Infra-red spectroscopy (FTIR): When investigating the graphs in Figs. 14-19 and the peaks resulted from existent functional groups in samples, no significant change is spotted such that the stretching absorption of $\mathrm{CH}$ in $3000-2850 \mathrm{~cm}^{-1}$ regions, the absorption of ketone in regions of $1740-1720 \mathrm{~cm}^{-1}$ and that of sulphates in 3000-2850 $\mathrm{cm}^{-1}$ regions, were equal for all dyed samples, untreated or treated with plasma. But, a low absorption was observed in $2350 \mathrm{~cm}^{-1}$ region pertinent to alkene or nitrile. As the operation time of plasma increases, the peak intensity increases too. This way, it can be concluded that the fabrics dye fastness after washing stage is acceptable and satisfactory for these samples and in other words, the plasmatic conditions are not responsible
TABLE-5

COMPARISON BETWEEN $\triangle E$ AND CIE TRIPLE-STIMULI OF SAMPLES DYED WITH YELLOW 105 AND PLASMA TREATED REACTIVE AFTER WASHING

Dyed with $\% 1$ of reactive yellow 105 after washing

\begin{tabular}{cccc}
\hline Time (plasma) & Untreated & $1 \mathrm{~min}$ & $5 \mathrm{~min}$ \\
\hline$\lambda_{\max }(\mathrm{nm})$ & 370 & 370 & 370 \\
$\mathrm{~K} / \mathrm{S}$ & 3.02 & 2.8 & 2.53 \\
$\mathrm{~L}^{*}$ & 88.24 & 89.61 & 88.89 \\
$\mathrm{a}^{*}$ & -2.79 & 50.43 & 49.24 \\
$\mathrm{~b}^{*}$ & 54.29 & 93.36 & 93.89 \\
$\mathrm{C}^{*}$ & 54.36 & -2.95 & -3.32 \\
$\mathrm{~h}^{\circ}$ & 92.94 & 50.35 & 49.13 \\
$\Delta \mathrm{E}$ & - & 66.04 & 65.38 \\
\hline
\end{tabular}

\section{TABLE-6}

COMPARISON BETWEEN $\triangle$ E AND CIE TRIPLE-STIMULI OF SAMPLES DYED WITH YELLOW 3 AND PLASMA TREATED REACTIVE AFTER WASHING

Dyed with \% 2 of Reactive yellow 3 after washing

\begin{tabular}{cccc}
\hline Time (plasma) & Untreated & $1 \mathrm{~min}$ & $5 \mathrm{~min}$ \\
\hline$\lambda_{\max }(\mathrm{nm})$ & 410 & 410 & 410 \\
$\mathrm{~K} / \mathrm{S}$ & 5.28 & 4.79 & 4.46 \\
$\mathrm{~L}^{*}$ & 80.51 & 80.54 & 80.89 \\
$\mathrm{a}^{*}$ & 13.12 & 66.76 & 65.74 \\
$\mathrm{~b}^{*}$ & 67.59 & 78.4 & 78.63 \\
$\mathrm{C}^{*}$ & 68.85 & 13.42 & 12.96 \\
$\mathrm{~h}^{\circ}$ & 79.01 & 65.4 & 64.45 \\
$\Delta \mathrm{E}$ & - & 54.72 & 53.76 \\
\hline
\end{tabular}

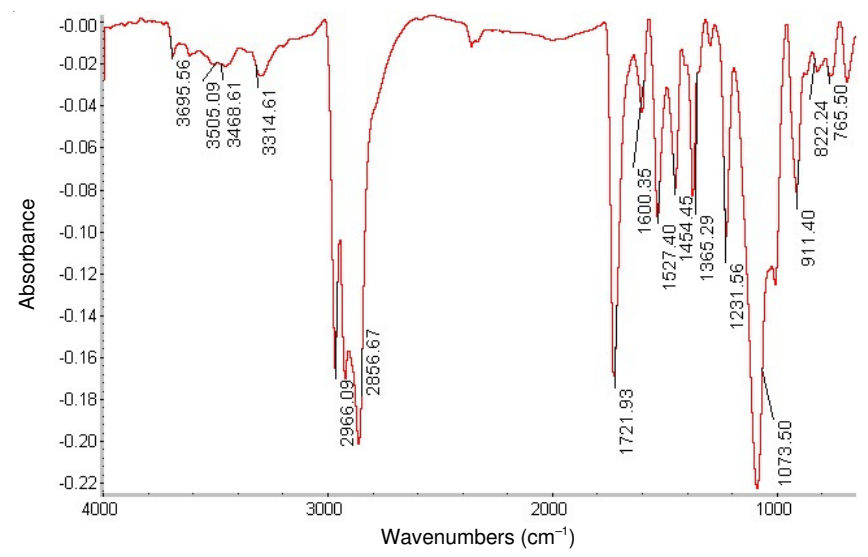

Fig. 14. FTIR graph of sample dyed with untreated reactive yellow 105

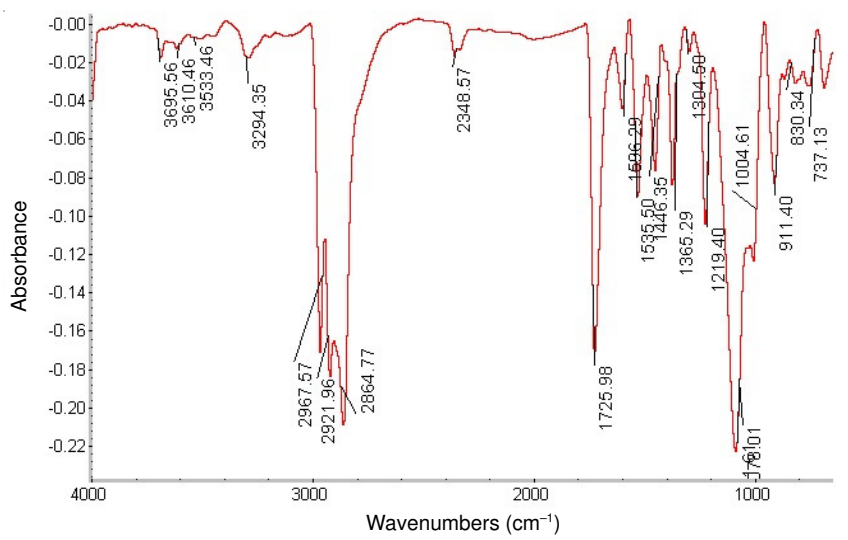

Fig. 15. FTIR graph of sample dyed with plasma treated reactive yellow 105 at 1 min of plasma exposure time 


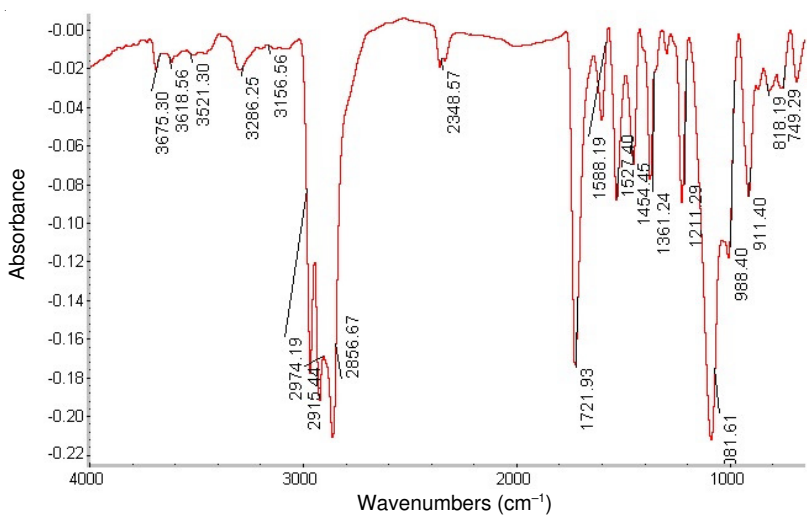

Fig. 16. FTIR graph of sample dyed with plasma treated reactive yellow 105 at 5 min of plasma exposure time

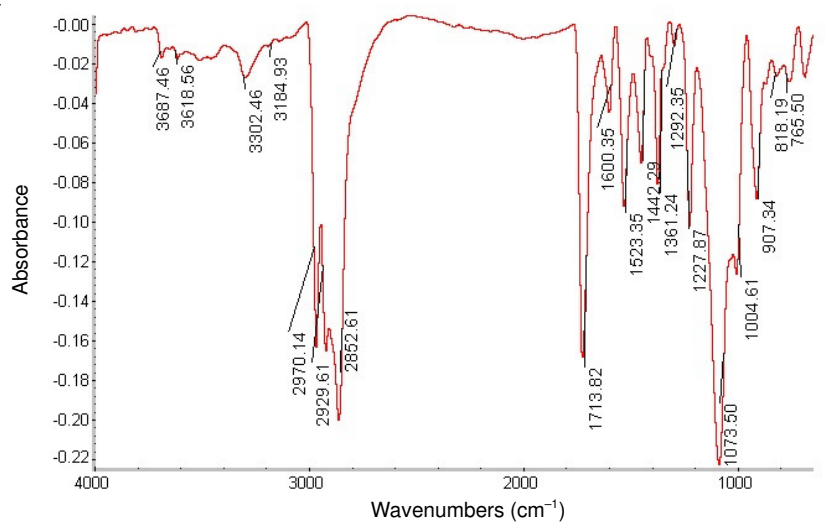

Fig. 17. FTIR graph of sample dyed with untreated reactive yellow 3

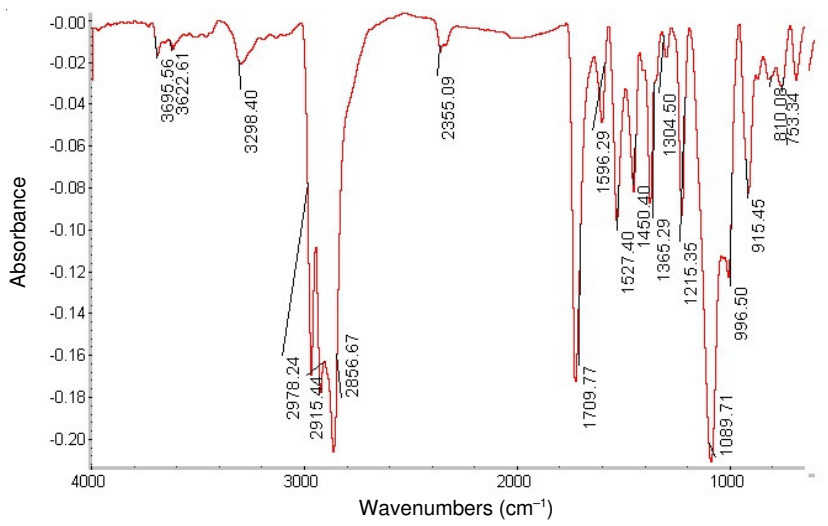

Fig. 18. FTIR graph of sample dyed with plasma treated reactive yellow 3 at $1 \mathrm{~min}$ of plasma exposure time

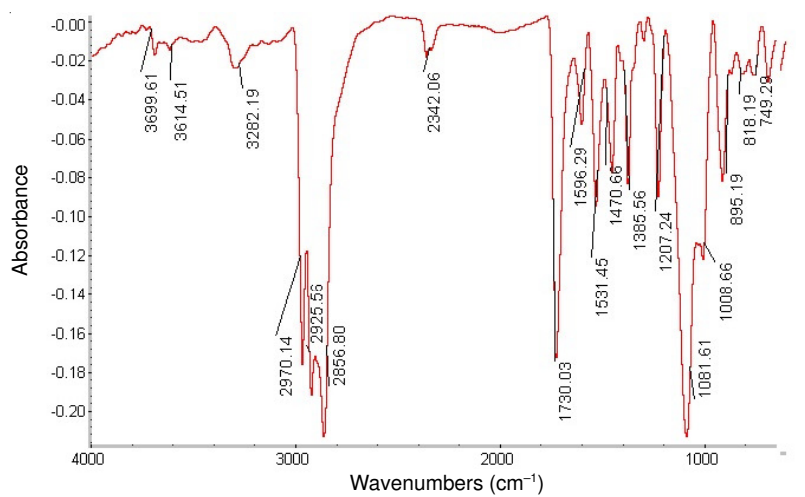

Fig. 19. FTIR graph of sample dyed with plasma treated reactive yellow 3 at $5 \mathrm{~min}$ of plasma exposure time for the failure in creation of washing fastness in the fabric compared to the untreated one. In fact, this shows the insignificant influence of this operation on the reflective spectrum and K/S values.

\section{Conclusion}

In this research, the bleached cotton fabric sample was dyed using reactive yellow 3 and reactive yellow 105 dyes and then the dyed samples were exposed to plasma condition of nitrogen gas at times of 1 and 5 min and in the next stages, the effect of plasma on destruction of surface morphology were investigated and evaluated using scanning electronic microscope with magnification power of 3000 . The results obtained from plasmatic conditions in determining K/S, b*, a*, $\mathrm{L}^{*}$ and $\mathrm{R} \%$ rates, studied using reflective spectrophotometer machine, show that samples dyed with both types of reactive dyes enjoy dye fastness; however, the washing fastness has partially decreased after cold plasma operation which can be ignored, as ATR-FTIR test proves it. Also, the images taken from plasma effect on surface morphology using SEM, shows that the increase in nitrogen gas plasma operation time will result in increase of destruction and this is more visible in samples dyed with reactive yellow 3 .

\section{REFERENCES}

1. V. Alenka, M. Miran, S. Simona, P. Zdenka, S. Karin and H. Nina, Sci. Vacuum, 30, 1 (2009).

2. J. Kang, AATCC Rev, 28, (2004).

3. J. Kang, AATCC Rev., 29, (2004).

4. J. Larrieu, F. Clement, B. Held, N. Soulem, F. Luthon and C. Guimon, Surf. Interface Anal., 37, 544 (2005).

5. M.R. Wertheimer, A.C. Fozza and A. Hollander, Nucl. Instrum. Meth. $B, \mathbf{6 5}, 151$ (1999)

8. L.J. Gerenser, in eds.: D.A. Glocker, S.I. Shah, W.D. Westwood, Handbook of Thin Film Process Technology, Bristol: IOP (1998).

9. H.U. Poll, U. Schladitz and S. Schreiter, Surf. Coat. Technol., 489, 142 (2001).

6. C. Zhang and K. Fang, Sci. Surf. Coat. Technol., 203, 2058 (2009).

7. C.W.M. Yuen, S. Jiang, C. Kan and W. Tung, Appl. Surf. Sci., 253, 5250 (2007).

10. M.G. McCord, Y.J. Hwang, P.J. Hauser and Y. Qiu, Tex. Res. J., 72, 491 (2002).

11. Z.S. Cai, Y.P. Qui, C.Y. Zhang, Y.J. Hwang and M. McCord, Tex. Res. J., 73, 670 (2003).

12. K. Fang, S. Wang, C. Wang and A. Tian, J. Appl. Polym. Sci., 107, 2949 (2008).

13. C.W. Kan, C.W. Yuen, Nucl. Instrum. Methods Phys. Res. B, 266, 127 (2008).

14. C. Kan, K. Chan and Y. Marcus, Autex. Res. J., 3, (2003).

15. C.W. Kan, K. Chan, C.W.M. Yuen and M.H. Miao, J. Mater. Process. Technol., 82, 122 (1998).

16. C.W. Kan, K. Chan, C.W.M. Yuen and M.H. Miao, J. Mater. Process. Technol., 83, 180 (1998). 